\title{
Erratum to: Is the derivative a function? If so, how do we teach it?
}

\author{
Jungeun Park 1
}

Published online: 16 August 2015

(C) Springer Science+Business Media Dordrecht 2015

\section{Erratum to: Educ Stud Math (2015) 89:233-250 \\ DOI 10.1007/s10649-015-9601-7}

The original version of this article contained two errors in Section 4.1.2 (p. 243). The corrected version is given below.

First, the instructors' word use did not explicitly indicate the object on which the limit operated when illustrating the limit process for defining the derivative or determining where the derivative exists. They all used "slope(s)" with "secant line(s)," but did not use "slope" with "tangent line" in most cases. Alan's explanation of Fig. 2b was:

The average rate of change is the green one that's the slope of the secant line [showing one secant line]...Getting closer and closer for $h$ to get into zero, we got really closer [showing more secant lines for each $h$ value], h got smaller, even smaller until we get $h$ going to zero, so we get the tangent line [showing the tangent line]. (Alan, 09-14-2009, Italics added)

The online version of the original article can be found at http://dx.doi.org/10.1007/s10649-015-9601-7.

Jungeun Park

Jungeun@udel.edu

1 Department of Mathematical Sciences, University of Delaware, 516 Ewing Hall, Newark, DE 19716, USA 